\title{
Modelling cost-effectiveness of tenofovir for prevention of mother to child transmission of hepatitis B virus (HBV) infection in South Africa
}

Jolynne Mokaya ${ }^{1}$, Edward A. O. Burn², Cynthia Raissa Tamandjou ${ }^{3}$, Dominique Goedhals ${ }^{4}$, Eleanor J. Barnes ${ }^{1,5,6}$, Monique Andersson ${ }^{3,7}$, Rafael Pinedo-Villanueva ${ }^{2}$ and Philippa C. Matthews ${ }^{1,6,7^{*}}$ (D)

\begin{abstract}
Background: International sustainable development goals for the elimination of viral hepatitis as a public health problem by 2030 highlight the need to optimize strategies for prevention, diagnosis and treatment of hepatitis B virus (HBV) infection. An important priority for Africa is to have affordable, accessible and sustainable prevention of mother to child transmission (PMTCT) programmes, delivering screening and treatment for antenatal women and implementing timely administration of HBV vaccine for their babies.

Methods: We developed a decision-analytic model simulating 10,000 singleton pregnancies to assess the costeffectiveness of three possible strategies for deployment of tenofovir in pregnancy, in combination with routine infant vaccination: S1: no screening nor antiviral therapy; S2: screening and antiviral prophylaxis for all women who test HBsAg-positive; S3: screening for HBsAg, followed by HBeAg testing and antiviral prophylaxis for women who are HBsAg-positive and HBeAg-positive. Our outcome was cost per infant HBV infection avoided and the analysis followed a healthcare perspective.

Results: Based on 10,000 pregnancies, S1 predicts 45 infants would be HBV-infected at six months of age, compared to 21 and 28 infants in S2 and S3, respectively. Relative to S1, S2 had an incremental cost of \$3940 per infection avoided. $\mathrm{S} 3$ led to more infections and higher costs.

Conclusion: Given the long-term health burden for individuals and economic burden for society associated with chronic HBV infection, screening pregnant women and providing tenofovir for all who test HBsAg+ may be a costeffective strategy for South Africa and other low/middle income settings.
\end{abstract}

Keywords: Tenofovir, Hepatitis B, HBV, PMTCT, Elimination, Transmission, Epidemiology, Africa

\section{Background}

In order to meet targets set by international Sustainable Development Goals for the elimination of Hepatitis B Virus (HBV) infection as a public health problem by the year 2030 [1], enhanced efforts are required to reduce

* Correspondence: philippa.matthews@ndm.ox.ac.uk

${ }^{1}$ Nuffield Department of Medicine, Medawar Building, South Parks Road, Oxford OX1 3SY, UK

${ }^{6}$ National Institute of Health Research, Oxford Biomedical Research Centre, John Radcliffe Hospital, Headley Way, Headington, Oxford OX3 9DU, UK Full list of author information is available at the end of the article the incidence of new cases. Strategies that set out to achieve this need to be carefully evaluated, both on the grounds of effect on individual and population health, and also based on value for money. HBV infection is endemic in many low/middle income settings [2-4], where economic evaluations are particularly important for informing the appropriate deployment of limited health care resources.

Prevention of mother to child transmission (PMTCT) is a cornerstone of HBV elimination strategies. Reducing vertical transmission is crucial to population health, as up to $90 \%$ of neonates who are exposed to HBV

(c) The Author(s). 2019 Open Access This article is distributed under the terms of the Creative Commons Attribution 4.0 International License (http://creativecommons.org/licenses/by/4.0/), which permits unrestricted use, distribution, and reproduction in any medium, provided you give appropriate credit to the original author(s) and the source, provide a link to the Creative Commons license, and indicate if changes were made. The Creative Commons Public Domain Dedication waiver (http://creativecommons.org/publicdomain/zero/1.0/) applies to the data made available in this article, unless otherwise stated. 
perinatally become chronic carriers, compared to only $5 \%$ of those exposed as adults $[5,6]$. Current recommended practice includes screening antenatal women for infection using hepatitis B surface antigen (HBsAg), and risk-stratification based on further laboratory tests for hepatitis B e-Antigen (HBeAg) and/or HBV DNA viral load (VL), which can be used to stratify the risk of vertical transmission. PMTCT guidelines suggest administering a three dose $\mathrm{HBV}$ vaccine to all infants with the first dose administered within $24 \mathrm{~h}$ of birth and two other doses provided at 6 and 10 weeks respectively, as well as administering hepatitis B immunoglobulin (HBIg) to high risk babies in the first day of life [7, 8]. Together, these strategies reduce the rate of vertical transmission by $85-95 \%$ [9], representing a crucial component of efforts towards HBV elimination [10-12]. However, breakthrough transmission can occur, especially among mothers with high HBV VL [13], in settings where the first dose of vaccine is delayed, and where HBIg is not available. The use of antivirals during pregnancy can therefore be included as an additional measure to decrease transmission risk [14]. Tenofovir disoproxil fumarate (TDF) has a track record of safety and efficacy in this setting $[15,16]$, and is included in some guidelines for HBV PMTCT $[8,17]$.

$\mathrm{HBV}$ is endemic in Africa, with a prevalence of $>8 \%$ in many populations $[18,19]$. The risk of MTCT in this continent is enhanced by lack of routine antenatal screening $[20,21]$, deliveries taking place outside healthcare facilities, delayed first dose of HBV vaccine until age six weeks by many vaccine programmes, and inadequate access to TDF and HBIg $[18,20]$. A metaanalysis of data from sub-Saharan Africa (sSA) demonstrated a perinatal transmission rate of $38 \%$ among women who tested positive for $\mathrm{HBsAg}$ and $\mathrm{HBeAg}$, in the absence of any PMTCT interventions [22]. An important priority for Africa is to have affordable, accessible and sustainable PMTCT programmes that deliver screening and treatment for antenatal mothers, and oversee timely administration of HBV birth vaccine for their babies [10, 23]. Providing antiviral treatment for HBV PMTCT relies on HBV diagnosis, but currently there is not widespread access to laboratory-based assays for HBsAg, HBeAg and HBV DNA viral load, and many antenatal programmes do not provide routine $\mathrm{HBV}$ screening.

In order to identify the most cost-effective approach to HBV PMTCT we have evaluated antenatal screening for HBV infection using standard laboratory assays for HBsAg and treating HBsAg-positive women with TDF, based on South Africa as a model situation. In recognising the important role that lateral flow assays can play in point of care testing (POCT) for HBsAg, we also assessed the cost effectiveness of this approach to diagnostic screening [21,
24]. In South Africa, the majority of pregnant women are not routinely screened for HBV infection, vaccination begins at six weeks of age and HBIg is not available. Modelling the cost-effectiveness of HBV PMTCT interventions allows for a combined analysis of clinical outcomes and potential budget impact, which is fundamental to inform financial investment in HBV elimination programmes in sSA countries.

\section{Methods}

\section{Target population and study perspective}

We used a hypothetical cohort of 10,000 pregnant South African women to evaluate the cost-effectiveness of three different strategies for antiviral therapy with TDF:

\section{Strategy 1 (S1): No TDF prophylaxis}

No pregnant woman is screened for HBsAg and therefore no HBV treatment is given perinatally. To date, this is the situation in many resource-limited settings in sSA.

\section{Strategy 2 (S2): Screening and TDF prophylaxis for all women who test $\mathrm{HBs} A g$ positive}

All pregnant women are screened for HBsAg; those who test positive are treated with TDF from 28 weeks gestation to four weeks post-partum.

\section{Strategy 3 (S3): Screening and TDF prophylaxis for women} who are both $\mathrm{HBsAg}$ positive and $\mathrm{HBeAg}$ positive

All pregnant women are screened for HBsAg; those who test positive are then screened for HBeAg. Only those who are $\mathrm{HBeAg}$ positive are treated with TDF from 28 weeks gestation to four weeks post-partum.

We populated our model with data using a healthcare system perspective and therefore only considered costs that would be directly incurred by the Department of Health (DOH). These costs relate to screening and treatment of HBV infection during pregnancy. Individual patient information was not included in this analysis. Ethics approval was not required for this study.

\section{Assumptions}

For the purposes of this analysis, we made a number of modelling assumptions:

- All pregnancies are singleton and result in the delivery of a live infant;

- All infants receive HBV vaccine at 6,10 and 14 weeks, as per standard practice to date in South Africa. Even for babies born to mothers who are $\mathrm{HBsAg+}$, birth dose vaccination is not offered within 24h (due to lack of maternal screening);

- HBV screening uptake is $100 \%$ among antenatal women; 
- $\mathrm{HBsAg}$ and $\mathrm{HBeAg}$ are tested using laboratory assays which are $100 \%$ sensitive and specific;

- Infants born to mothers who were HBsAg negative were assumed also to be HBsAg negative;

- We made assumptions around our point estimates due to lack of data.

\section{Theoretic modelling approach}

A decision tree providing a framework for comparison of the three strategies is shown in Fig. 1. The decision tree considers the period covering weeks 28 through 40 of gestation up to six months post-partum. Model structure was the same for all strategies. HBsAg-positive mothers had a defined probability of being HBeAg positive or negative; these probabilities were the same for all strategies. Children whose mothers were HBsAg positive had a probability of being HBsAg positive themselves (i.e. a case of MTCT). This probability depended on whether the mother was also HBeAg positive and whether antiviral prophylaxis was received by the mother during pregnancy, which varied between strategies.

\section{Measurement of effectiveness}

Table 1 shows parameters used in our model. We searched the published literature, and identified four studies from within the last six years that reported the prevalence of HBV infection among pregnant women in South Africa [21, 25-27]. To calculate prevalence of HBV infection, we combined the total number of women from each study who tested HBsAg-positive and divided these by the total number of individuals included in these cohorts. From these pooled data, the overall prevalence of HBsAg among pregnant women was 3.6\% (129/3614). HBeAg status was reported for 126 of these 129; the prevalence of $\mathrm{HBeAg}$ among $\mathrm{HBsAg}+$ women was 29/126 (23\%).

We derived point estimates for perinatal transmission with and without antiviral therapy during pregnancy from a published systematic review and a meta-analysis $[15,22]$. In the absence of intervention (HBV vaccination, HBIg and/or antiviral therapy), the perinatal transmission rate for $\mathrm{HBsAg}+/ \mathrm{HBeAg}+$ mothers in sSA was estimated at $38 \%$, and for $\mathrm{HBsAg}+/ \mathrm{HBeAg}$ - mothers was $4.8 \%$ [22]. Due to lack of data, we used these estimates in our model, although we assumed that infants were vaccinated starting at the age of six weeks.

There is only one randomised control trial that examines the efficacy of TDF on HBV PMTCT, in which perinatal TDF reduced the risk of infant HBsAg seropositivity by $71 \%$ [14]. However, data from a systematic review and meta-analysis estimate this figure as 77\% [15]. We reduced the perinatal transmission rate for women receiving no

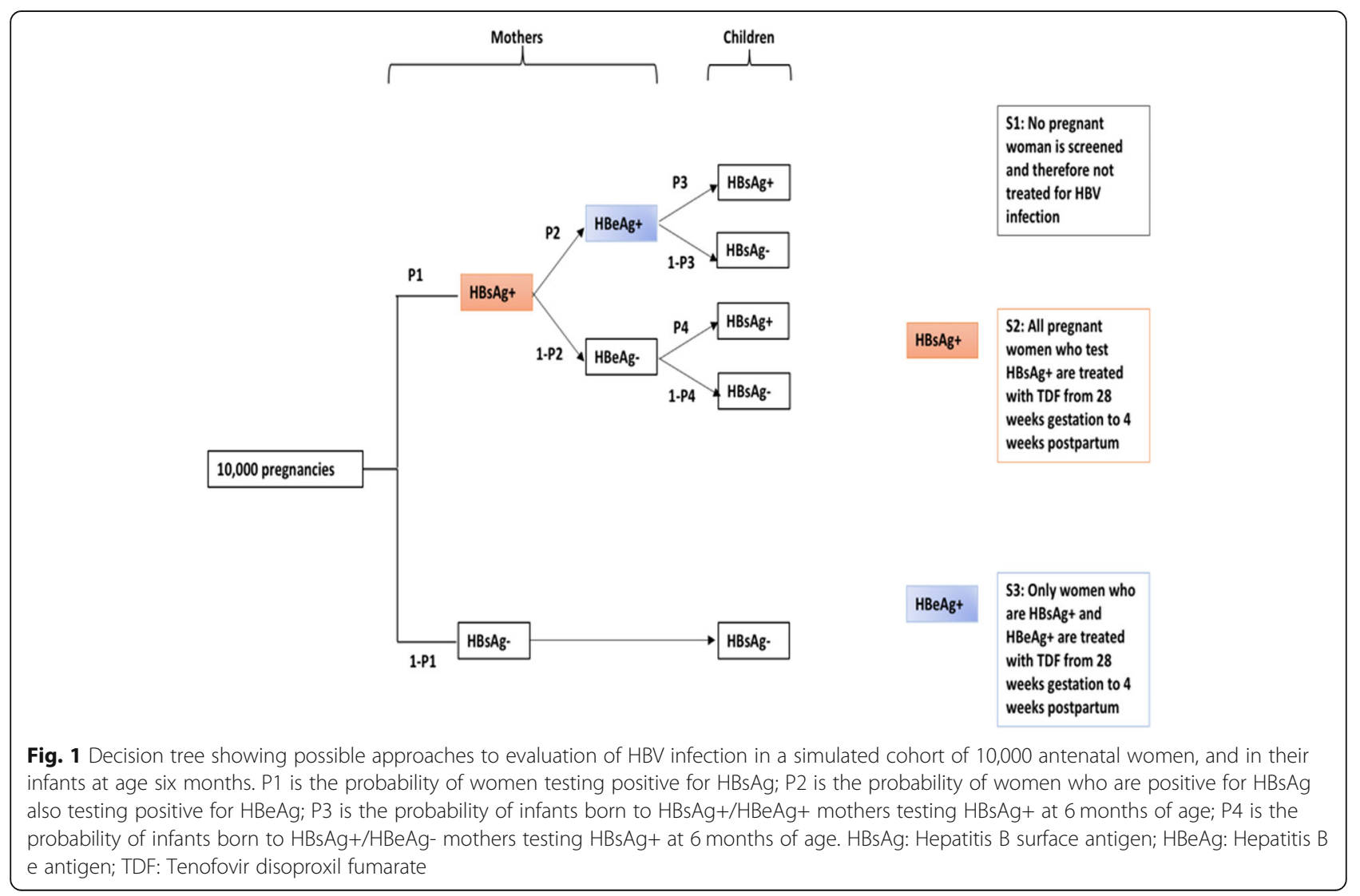


Table 1 Parameters used for input into a model of cost-effectiveness of tenofovir for PMTCT in South Africa, based on a simulated cohort of 10,000 antenatal women and their babies

\begin{tabular}{|c|c|c|c|}
\hline Parameter & Point estimate & $\begin{array}{l}\text { Uncertainty (Lower } \\
\text { bound - Upper bound) }\end{array}$ & $\begin{array}{l}\text { Source } \\
\text { (references) }\end{array}$ \\
\hline p1: Probability of mother being $\mathrm{HBsAg}+$ & $3.6 \%$ & $3.1-7.4 \%^{b}$ & {$[21,25-27]$} \\
\hline p2: Probability of mother who is $\mathrm{HBsAg}+$ being $\mathrm{HBeAg}+$ & $23 \%$ & $16.7-42.9 \%^{\mathrm{b}}$ & {$[21,25-27]$} \\
\hline $\begin{array}{l}\text { p3: Probability of mother who is HBsAg+ and HBeAg+ having } \\
\text { child who is HBsAg+ (no PMTCT) }\end{array}$ & $38.3 \%$ & $7.0-74.4 \%^{\mathrm{b}}$ & [22] \\
\hline $\begin{array}{l}\text { p4: Probability of mother who is HBsAg+ and HBeAg- having } \\
\text { child who is HBsAg+ (no PMTCT) }\end{array}$ & $4.8 \%$ & $0.1-13.3 \%^{b}$ & {$[22]$} \\
\hline Relative risk reduction (efficacy) of TDF & $71 \%$ & $26-89 \%$ & {$[15]$} \\
\hline Antiviral adherence & $73.5 \%$ & $69.3-77.5 \%$ & {$[28]$} \\
\hline S2: Cost of diagnostics: laboratory test for $\mathrm{HBsAg}$ & $\$ 9.1$ per mother & & {$[29]$} \\
\hline S3: Cost of diagnostics: laboratory test for HBsAg and HBeAg & $\begin{array}{l}\$ 9.1 \text { per mother (HBsAg test) }+\$ 9.1 \text { for } \\
\text { HBsAg+ mother } \\
\text { (HBeAg test) }\end{array}$ & & {$[29]$} \\
\hline S2: Cost of diagnostics: POCT for HBsAg & $\$ 2 *$ all mothers & & {$[29]$} \\
\hline $\begin{array}{l}\text { S3: Cost of diagnostics: POCT for HBsAg+ and laboratory test for } \\
\text { HBeAg+ }\end{array}$ & $\begin{array}{l}\$ 2 * \text { for all mothers (HBsAg test) }+\$ 9.1 * \\
\text { for all HBsAg+ mothers (HBeAg test) }\end{array}$ & & {$[29]$} \\
\hline $\begin{array}{l}\text { Treatment cost: monthly cost of TDF, applied to all HBsAg+ } \\
\text { women (S2) or only to } \mathrm{HBsAg}+/ \mathrm{HBeAg}+\text { women (S3) }\end{array}$ & $\$ 2.48^{\mathrm{a}} /$ month & & {$[30]$} \\
\hline POCT sensitivity & $97.6 \%$ & & {$[21]$} \\
\hline $\begin{array}{l}\text { Estimated relative risk reduction (efficacy) of TDF when } \\
\text { combined with birth dose vaccine and HBlg }\end{array}$ & $90 \%$ & $85-95 \%$ & {$[9]$} \\
\hline Prevalence of TDF resistance & $0.08 \%$ & & {$[31]$} \\
\hline
\end{tabular}

${ }^{a}$ To cover the cost of TDF treatment from 28 weeks' gestation to 4 weeks post-delivery, we multiplied the cost for one month by four. A triangular distribution allows for uncertainty in all probabilities (except cost which we assume is fixed)

${ }^{\mathrm{b}}$ The uncertainty values for $\mathrm{p} 1$ and $\mathrm{p} 2$ were derived from studies that had the lowest and highest HBsAg prevalence rates, representing lower and higher bounds respectively; whereas uncertainly values for P3 and P4 were from 95\% confidence intervals around the mean value

TDF by $71 \%$ to obtain a point estimate for perinatal transmission rate for those receiving TDF peripartum prophylaxis; these were estimated at $11.1 \%$ for $\mathrm{HBeAg}$-positive women and $1.4 \%$ for HBeAg-negative women. We accounted for uncertainty around point estimates, as the original study was conducted in China and with a different protocol (combining TDF during pregnancy in combination with birth dose vaccine and $\mathrm{HBIg}$ ).

Due to lack of data on HBV antiviral adherence rates among pregnant women in sSA, we used HIV antiretroviral adherence rate among women during and after pregnancy, obtained from a published systematic literature review which included studies from low-income, middle-income, and high-income countries [28]; this is estimated at $73.5 \%$. We incorporated an expected adherence of $73.5 \%$ into the model, assuming that those mothers who didn't adhere would incur the full cost of treatment but would have the same probability of transmission as untreated women.

\section{Outcomes}

Our outcome was the cost per HBV infection avoided among infants at the age of six months. This outcome was selected because it is quantifiable, clinically relevant and could be easily compared between strategies.

\section{Estimating costs}

We derived costs for laboratory assays for HBsAg and $\mathrm{HBeAg}$, and POCT, from a 2015 price list produced by $\mathrm{Na}-$ tional Health Laboratory Services (NHLS) and DOH, South Africa [29]. We used the cost of TDF from International Medical products price guide for year 2015 [30]. We converted the price of HBsAg laboratory assay (ZAR108.86), HBsAg POCT (ZAR18), HBeAg laboratory assay (ZAR108.86) and HBV DNA test (R1173.32) from South African Rand (ZAR) to US Dollars (USD); 1 ZAR = 0.083 USD as at 1st May 2018. HBsAg screening cost applied to all women in S2 and S3; HBeAg screening costs applied to women who tested positive for HBsAg in S3. TDF costs applied to all women who tested positive for $\mathrm{HBsAg}$ in $\mathrm{S} 2$, and women who tested positive for both HBsAg and HBeAg in S3.

\section{Scenario analysis}

We assessed the impact of three additional scenarios, estimating the number of MTCT cases, costs and incremental cost per infection avoided in each case:

(i.) Antenatal screening for $\mathrm{HBV}$ infection using a rapid point of care test (POCT) 
We estimated the cost-effectiveness of using a POCT for HBsAg screening, based on a POCT sensitivity of $97.6 \%$ and specificity of $100 \%$, compared to laboratory ELISA HBsAg testing as the gold standard [21]. We incorporated this into the model by reassigning the proportion of women expected to test falsely $\mathrm{HBsAg-}$ negative using a POCT into the untreated group and removing the cost of TDF treatment for these individuals.

(ii.) Recommended PMTCT guidelines: Universal birth dose vaccination and HBIg

To assess the cost-effectiveness of antenatal screening and treating of $\mathrm{HBsAg}$-positive women with TDF in combination with universal birth dose vaccine and $\mathrm{HBIg}$, we estimated that perinatal transmission rate will be reduced by $90 \%$ [9]. To incorporate the impact of TDF in our cohort, we therefore reduced the perinatal transmission rate by $90 \%$; MTCT rates were thus estimated for $\mathrm{HBsAg}+$ women who are $\mathrm{HBeAg}+$ and $\mathrm{HBeAg}$ - at 3.8 and $0.48 \%$ respectively.

\section{(iii.) HBV resistance to TDF}

In order to consider the possible impact of drug resistance on the PMTCT strategies outlined here, we estimated the prevalence of TDF resistance based on HBV sequences retrieved from the HBV database (https:// hbvdb.ibcp.fr/HBVdb/) [32] accessed on the 18th April 2018. The numerator was the total number of sequences with mutations associated with reduced sensitivity/resistance to TDF (rtA194T, rtN236T, rtS78T) [31, 33], and the denominator was the total number of sequences available in the database $(49 / 6287,0.8 \%)$. This prevalence was incorporated into the model by re-assigning $0.8 \%$ of those receiving TDF into the untreated category (as although they receive the drug, resistance would render this functionally equivalent to no treatment).

\section{Analytic methods and definitions}

We used the following definitions:

- A strategy was 'dominated' if it had a higher expected cost and more predicted cases of HBV MTCT compared to an alternative strategy.

- The incremental cost per HBV infection avoided was determined by dividing the difference in cost between two strategies over the difference in number of infections.

We compared the strategies by first applying decision rules to eliminate any strategies which were dominated by others, and then estimated the incremental cost per infection avoided for the remaining strategies.
The combined effect of uncertainty in probability estimates was assessed using probabilistic sensitivity analysis, with 1000 Monte Carlo simulations run. We used triangular distributions to approximate Beta distributions for probabilities. Triangular distributions required a lower and upper bound, with the peak taken as the expected (mean) value. The impact of uncertainty is shown on a cost-effectiveness plane, where sets of incremental costs and number of infections avoided are plotted for S2 and S3, relative to S1.

\section{Standardised criteria}

Our analysis conforms to the standardised criteria for economic evaluations of health interventions. Our CHEERS checklist can be reviewed as Additional file 1: Table S1 on-line: https://doi.org/10.6084/m9.figshare.7265582.v2

\section{Results}

Cost-effectiveness of strategies for HBV PMTCT under base case assumptions

We modelled three different approaches to peri-partum TDF prophylaxis: S1: no screening or antiviral therapy; S2: screening and antiviral prophylaxis for all women who test $\mathrm{HBsAg}$-positive; S3: screening for $\mathrm{HBsAg}$, followed by HBeAg testing and antiviral prophylaxis for women who are $\mathrm{HBsAg}$-positive and $\mathrm{HBeAg}$-positive (Fig. 1). Based on the simulated scenario of 10,000 singleton pregnancies in South Africa, with no antiviral therapy for HBV PMTCT (S1), and using a laboratorybased assay for HBsAg detection, our model predicts 45 infants would be infected with HBV at age six months (incidence $0.45 \%$ ). If antiviral prophylaxis interventions are employed based on S2 and S3, this would be reduced to 21 and 28 infants, (incidence 0.21 and $0.28 \%$ ) respectively, at the cost of deploying the intervention for the whole population. Compared to S1, cost per infection avoided was $\$ 3940$ for S2, lower than for S3 which would be more costly and avoid a lower number of infections (Table 2; Fig. 2).

\section{Cost-effectiveness of strategies for HBV PMTCT under scenario analyses}

(i.) Antenatal screening for HBV infection using a rapid point of care test (POCT)

POCT for HBsAg was cheaper in comparison to laboratory-based HBsAg assay (for S2: POCT \$23,401 vs lab test $\$ 94,571$ and for S3: POCT $\$ 23,979$ vs lab test \$95,097); (Tables 2 and 3). Based on reasonable assumptions about sensitivity of HBsAg detection via laboratory ELISA vs POCT (100 and 97.6\%, respectively [21]), from our simulated cohort, laboratory testing would detect all maternal HBV infections $(n=360$ in our hypothetical 
Table 2 Cost-effectiveness results under base case assumptions for screening for HBsAg using laboratory-based assay on a simulated birth cohort of 10,000 live singleton infants

\begin{tabular}{llll}
\hline Strategy & $\begin{array}{l}\text { Number of infant HBV } \\
\text { infections }^{\mathrm{a}}(95 \% \mathrm{Cl})\end{array}$ & $\begin{array}{l}\text { Cost of deploying the intervention for the } \\
\text { whole population }^{\mathrm{b}}(n=10,000) \text { in USD }(95 \% \mathrm{Cl})\end{array}$ & $\begin{array}{l}\text { Incremental cost per infection } \\
\text { avoided (USD) }\end{array}$ \\
\hline S1 & $45(29-121)$ & 0 & - \\
S2 & $21(14-69)$ & $94,571(94,487-95,509)$ & 3940 (compared to strategy S1) \\
S3 & $28(19-76)$ & $95,097(94,980-97,244)$ & Dominated $^{c}$ (by strategy S2) \\
\hline
\end{tabular}

${ }^{a}$ World Health Organisation (WHO) criteria for HBV elimination states an aim of $90 \%$ reduction in new chronic infection [1]

b Price of TDF estimated at \$2.48/month for strategies S2 and S3 [30]

c S3 is dominated due to both higher costs and higher infections compared to S2 $\mathrm{Cl}=$ confidence interval

cohort of 10,000), while the POCT approach would detect 351 and miss nine cases of infection. This loss of sensitivity translates into a marginal decrease in costeffectiveness: the incremental cost per infection avoided is estimated at $\$ 1017$ compared to $\$ 978$ if the test had $100 \%$ sensitivity.

(ii.) Recommended PMTCT guidelines: Universal birth dose vaccination and $\mathrm{HBIg}$

Incorporating universal birth dose vaccination and HBIg into our model, reduced the number of infants infected at six months and cost per infection avoided (S2: 15 infants infected; S3: 24 infants infected; and cost per infection avoided: \$3152), (Table 3), compared to when

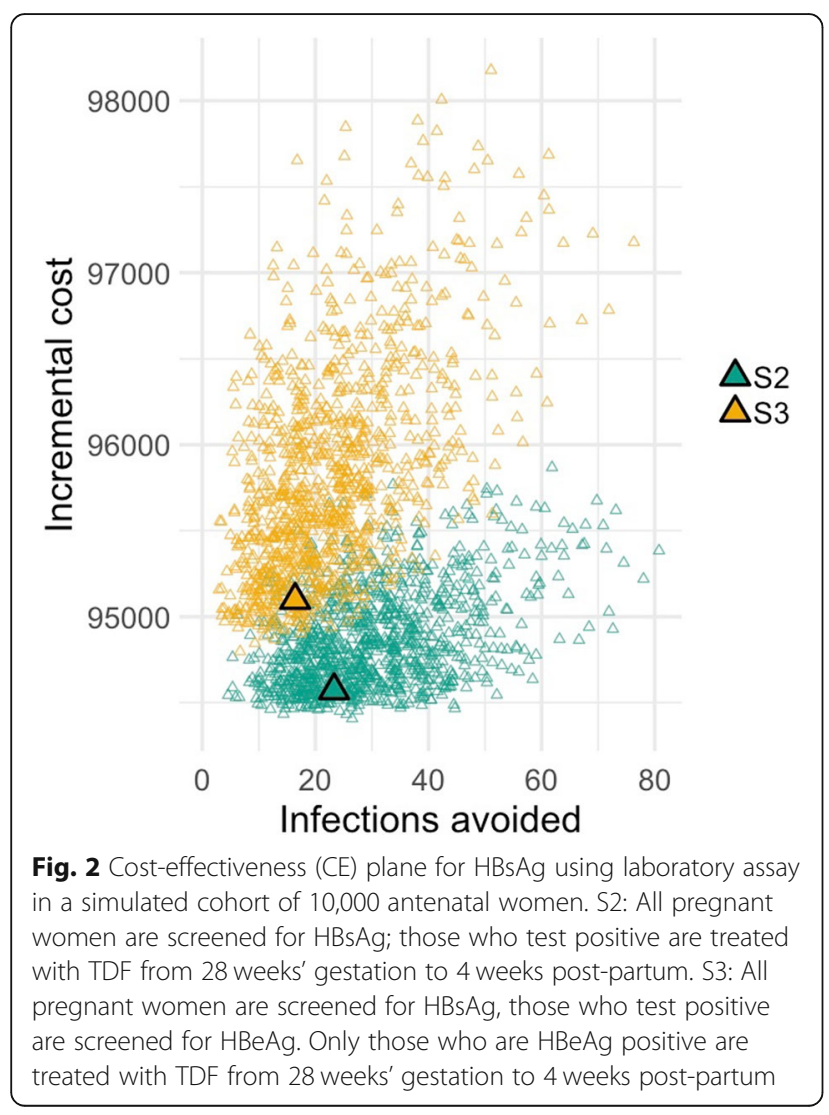

PMTCT intervention includes only TDF and HBV vaccination beginning at six weeks, (S2: 21 infants infected; S3: 28 infants infected; and cost per infection avoided: \$3940) (Table 2).

\section{(iii.) HBV resistance to TDF}

When we accounted for potential HBV resistance to TDF, based on a prevalence of drug resistance estimated at $0.8 \%$ [31], there was no change in incremental cost per infection avoided in $\mathrm{S} 2$.

\section{Data visualisation}

We reproduced our decision model as an R Shiny application (source code available here: https:/github.com/ edward-burn/PMTCT-HBV-cost-effectiveness-analysis).

This can be used to estimate cost-effectiveness of our three strategies using different input parameters. This will allow the analysis to be re-run for the same comparison of strategies but in contexts where the epidemiology of HBV infection, and costs of interventions, are different.

\section{Discussion}

Antenatal screening and treating of pregnant women for $\mathrm{HBV}$ is not routinely performed in many settings in sSA, despite the high population prevalence of HBV [21, 25-27]. In resource-limited settings, screening and providing prophylactic TDF for all pregnant women who are HBsAg positive may be the most efficient strategy, especially when using a POCT. These results are derived from a theoretical model, and careful consideration is needed for application to different real world settings.

Based on existing data for South Africa, screening and treating women with TDF from 28 weeks of pregnancy to four weeks post-delivery reduces the number of infants with HBV at six months. Previous studies have also reported on the safety and effectiveness of TDF for HBV PMTCT $[15,16]$. There is evidence for the costeffectiveness of combining antiviral therapy during pregnancy with HBV immunoprophylaxis, as highlighted by a systematic review and meta-analysis carried out in China [35] and a study conducted in North America 
Table 3 Cost-effectiveness results of different PMTCT strategies under scenario analyses on a simulated birth cohort of 10,000 live singleton infants

\begin{tabular}{|c|c|c|c|}
\hline Strategy & $\begin{array}{l}\text { Number of infant HBV } \\
\text { infections }^{\mathrm{a}}(95 \% \mathrm{Cl})\end{array}$ & $\begin{array}{l}\text { Cost of deploying the intervention for the } \\
\text { whole population }(n=10,000) \text { in USD }(95 \% \mathrm{Cl})\end{array}$ & $\begin{array}{l}\text { Incremental cost per infection } \\
\text { avoided (USD) }\end{array}$ \\
\hline \multicolumn{4}{|c|}{ Scenario analysis (i): POCT testing } \\
\hline S1 & $45(28-119)$ & 0 & \\
\hline $\mathrm{S} 2^{\mathrm{b}}$ & $22(14-73)$ & $23,401(23,330-24,243)$ & 1017 (compared to strategy S1) \\
\hline$S 3^{b}$ & $28(19-78)$ & $23,979(23,882-25,937)$ & Dominated $^{c}$ (by strategy S2) \\
\hline \multicolumn{4}{|c|}{ Scenario analysis (ii): Universal birth dose vaccination } \\
\hline S1 & $45(28-123)$ & 0 & - \\
\hline$S 2^{d}$ & $15(11-82)$ & $94,571(94,489-95,503)$ & 3152 (compared to strategy S1) \\
\hline$S 3^{\mathrm{d}}$ & $24(17-84)$ & $95,097(94,981-97,291)$ & Dominated ${ }^{c}$ (by strategy S2) \\
\hline \multicolumn{4}{|c|}{ Scenario analysis (iii): TDF resistance } \\
\hline S1 & $45(29-119)$ & 0 & - \\
\hline$S 2^{d}$ & $21(14-71)$ & $94,571(94,496-95,515)$ & 3940 (compared to strategy S1) \\
\hline$S 3^{d}$ & $28(18-79)$ & $95,097(94,980-97,252)$ & Dominated $^{c}$ (by strategy S2) \\
\hline
\end{tabular}

[36]. Interestingly, a recent study in Thailand conflicted with these findings by reporting no significant benefit of maternal TDF for PMTCT [37]. The lack of benefit from TDF in this case may be because all infants in the Thai study received birth dose of both $\mathrm{HBIg}$ and $\mathrm{HBV}$ vaccine, followed by four additional doses of HBV vaccine; this enhanced immunoprophylaxis probably accounts for the low rate of HBV transmission even among mothers who did not receive TDF. However, it is difficult to apply any of these findings to sSA, which differs in having limited (or no) access to HBIg, gaps in vaccine coverage, and delays in the first dose of $\mathrm{HBV}$ vaccine (in South Africa to date, as in many other settings in sSA, this has been until age six weeks) [20].

In this study, we provide a head-to-head economic analysis of simulated screening for $\mathrm{HBsAg}$ alone vs. use of HBsAg in combination with risk stratification using $\mathrm{HBeAg}$. Limited resources and infrastructure have impeded antenatal diagnosis and treatment of HBV [34, 39], and stratification of HBsAg-positive antenatal women with HBV DNA level and/or HBeAg to determine eligibility for TDF incurs further cost. Given that TDF is becoming more accessible for HBV treatment in Africa [12, 40], and has a well-established safety record as a result of antenatal use in HIV infection, treating all HBsAg positive women should be safe and practical, as well as cost-effective. As this option is simple to implement, it is therefore also most likely to be successful.

Given the high prevalence of HBV in Africa [2, 3], POCT is an appealing route to increasing diagnosis and therefore access to treatment. Although POCT proves advantageous on cost grounds, it is less sensitive than laboratory assays. Furthermore, laboratory support remains key for monitoring response to treatment, identifying and monitoring drug resistance, and evaluating prognosis.

There is a potential risk that increasing population exposure to TDF may increase selection of resistance [38]. However, the genetic barrier to TDF resistance is high, and more data are needed to determine the prevalence and clinical significance of putative drug resistance mutations. When accounting for an estimated background rate of $\mathrm{HBV}$ resistance to TDF, we show no change in the cost per infection avoided. While we recognise that TDF resistance is not currently a significant clinical concern, the modelling approach we have developed allows drug resistance to be factored in.

Despite the potential for increased risk of HBV MTCT in $\mathrm{HIV} / \mathrm{HBV}$ coinfection there is evidence to show that HIV has very little effect on HBV interventions [41]. Since HIV guidelines now recommend commencement of antiretroviral treatment as soon as HIV diagnosis is made [42], the impact of HIV on HBV PMTCT is further reduced.

We used a simple model with only a single outcome measure, thus overlooking other possible risks/benefits of antiviral therapy, including side-effects, drug interactions, and rebound hepatitis after treatment cessation. However, extensive experience has led to the inclusion of TDF in first-line regimens for HIV, including in pregnant women. Our base values were obtained from published literature, but there are limited data for $\mathrm{HBV}$ 
epidemiology and transmission for most African populations; we have acknowledged the lack of certainty around certain parameters by including confidence intervals. HBV DNA testing is the gold standard approach to HBV diagnosis, but is often not available in resourcelimited settings; we therefore used $\mathrm{HBeAg}$ status as a surrogate marker to represent infectivity and high VL and assumed $100 \%$ sensitivity for laboratory-based assays for HBsAg and HBeAg. This does not account for occult infection and other false negatives and may lead to an overestimation of the cost-effectiveness of maternal TDF.

The assumption that the first dose of HBV vaccine is delayed until age six weeks is an over-simplification: if birth dose vaccine is given more widely or targeted for infants born to HBsAg-positive mothers, more new infections will be averted.

We used data for South Africa, but recognise that HBV prevalence is substantially higher in other sSA settings (frequently reported at $\geq 8 \%$, the WHO threshold for high endemicity $[18,19])$ and this may influence the cost-effectiveness of proposed interventions. The low prevalence rate that we derived from South Africa may be a reflection that many antenatal women themselves received HBV vaccination as infants (if born after 1995). To facilitate cost-effectiveness simulations in other settings where antenatal HBV prevalence might be higher, we have provided an on-line interactive tool.

The findings of this study are applicable to settings where the cost of screening and treatment is publicly funded; in situations where individuals meet the cost of their own screening and treatment, our results cannot be applied to informing public health strategy. Our analysis did not consider the need for healthcare and laboratory infrastructure to support our proposed interventions, costs of monitoring pregnant women during TDF treatment, costs for clinical visits and counselling for mothers testing HBsAg positive, leading to an underestimation of the total costs included in providing robust PMTCT. However, this is offset by the potentially very substantial lifelong costs of chronic HBV infection, both to individuals and to society, arising from morbidity and mortality typically affecting young and middle-aged adults $[4,5,34$, 43]. This is difficult to quantify but leads to a substantial burden of chronic disease with economic consequences for the health-care system, as well as imposing financial consequences on families and society [34].

\section{Conclusion}

We have developed a simple, theoretical model that allows us to estimate the impact of providing TDF to antenatal women in a lower/middle income country setting, either based on HBsAg status alone, or incorporating risk-stratification with $\mathrm{HBeAg}$. These strategies reflect safe, practical interventions that could reasonably be deployed in many settings. There remains an urgent need for more data to underpin the relevant epidemiology, risks of MTCT, and relative benefits of different interventions in settings across sSA. In order to drive progress towards 2030 elimination targets, sustained investment is required to drive improvements in clinical services, provide universal access to antenatal screening, improve education of the public and health-care workers, and underpin robust deployment of PMTCT interventions including vaccination and TDF therapy.

\section{Additional file}

Additional file 1: Consolidated Health Economic Evaluation Reporting Standards (CHEERS) checklist - Modelling cost-effectiveness of alternative strategies for prevention of mother to child transmission of hepatitis B virus infection in South Africa. Available on-line at https://doi.org/10.6084/ m9.figshare.7265582.v2. (DOC 68 kb)

\section{Abbreviations}

HBeAg: Hepatitis B e antigen; HBlg: Hepatitis B immunoglobulin; HBsAg: Hepatitis B surface antigen; HBV: Hepatitis B Virus; MTCT: Mother to child transmission; PMTCT: Prevention of mother to child transmission; POCT: Point of care test; sSA: Sub Saharan Africa; TDF: Tenofovir disoproxil fumarate; VL: Viral load

\section{Acknowledgements}

Not applicable.

\section{Authors' contributions}

Conceived the study: JM, MA, PCM, CRT. Literature review: JM. Assimilated data to feed into the model: JM, DG, PCM. Analysed the data: JM, EAOB, RPV, PCM. Wrote the manuscript: JM, EAOB, PCM. Revised the manuscript: JM, CRT, DG, EJB, MA, RPV. All authors have read and approved the manuscript.

\section{Funding}

JM is funded by a Leverhulme Mandela Rhodes Scholarship. PCM is funded by the Wellcome Trust, grant number 110110. EB is funded by the Medical Research Council UK, the Oxford NIHR Biomedical Research Centre and is an NIHR Senior Investigator. The views expressed in this article are those of the author and not necessarily those of the NHS, the NIHR, or the Department of Health.

Availability of data and materials

All data generated or analysed during this study are included in this published article (and its Supplementary Information files).

Ethics approval and consent to participate

Not applicable.

Consent for publication

Not applicable.

Competing interests

The authors declare that they have no competing interests.

\section{Author details}

${ }^{1}$ Nuffield Department of Medicine, Medawar Building, South Parks Road, Oxford OX1 3SY, UK. ${ }^{2}$ Centre for Statistics in Medicine, University of Oxford, Oxford OX3 7LD, UK. ${ }^{3}$ Division of Medical Virology, Faculty of Medicine and Health Sciences, Stellenbosch University, Francie van Zijl Drive, Tygerberg 8000 , Cape Town, Republic of South Africa. ${ }^{4}$ Division of Virology, University of the Free State/National Health Laboratory Service, PO Box 339(G23), Bloemfontein 9300, Republic of South Africa. ${ }^{5}$ Department of Hepatology, Oxford University Hospitals NHS Foundation Trust, John Radcliffe Hospital, Headley Way, Oxford OX3 9DU, UK. ${ }^{6}$ National Institute of Health Research, 
Oxford Biomedical Research Centre, John Radcliffe Hospital, Headley Way, Headington, Oxford OX3 9DU, UK. ${ }^{7}$ Department of Microbiology and Infectious Diseases, Oxford University Hospitals NHS Foundation Trust, John Radcliffe Hospital, Headley Way, Oxford OX3 9DU, UK.

\section{Received: 19 December 2018 Accepted: 31 May 2019}

Published online: 26 June 2019

\section{References}

1. WHO. Combating hepatitis B and C to reach elimination by 2030. Advocacy Brief. 2016. http://apps.who.int/iris/bitstream/handle/10665/206453/WHO_ HIV_2016.04_eng.pdf;jsessionid=34488ABA33BD359C8415A10205FBEC09? sequence $=1$.

2. WHO. Hepatitis B Fact sheet 2018. Available from: http://www.who.int/ news-room/fact-sheets/detail/hepatitis-b.

3. Razavi-Shearer $D$, et al. Global prevalence, treatment, and prevention of hepatitis B virus infection in 2016: a modelling study. Lancet Gastroenterol Hepatol. 2018;3(6):383-403.

4. GBD 2017 Causes of Death Collaborators. Global, regional, and national agesex-specific mortality for 282 causes of death in 195 countries and territories, 1980-2017: a systematic analysis for the Global Burden of Disease Study 2017. Lancet. 2018;392(10159):1736-88.

5. Lavanchy D. Hepatitis B virus epidemiology, disease burden, treatment, and current and emerging prevention and control measures. Viral Hepat. 2004; 11(2):97-107.

6. Yi P, Chen R, Huang $Y$, Zhou R-R, Fan X-G. Management of mother-to-child transmission of hepatitis B virus: propositions and challenges. J Clin Virol. 2016;77:32-9.

7. Ayoub WS, Cohen E. Hepatitis B Management in the Pregnant Patient: an update. J Clin Transl Hepatol. 2016;4(3):241-7.

8. Lampertico P, et al. EASL 2017 Clinical Practice Guidelines on the management of hepatitis B virus infection. J Hepatol. 2017;67(2):370-98. https:/easl.eu/publication/management-of-hepatitis-b-virus-infection/.

9. Nelson NP, Jamieson DJ, Murphy TV. Prevention of perinatal Hepatitis B virus transmission. J Pediatric Infect Dis Soc. 2014;3 Suppl 1(Suppl 1):S7-12

10. McNaughton A, et al. Can we meet global challenges for elimination of Hepatitis B virus infection by 2030? Vaccine-mediated immunity in a south African cohort and a model of transmission and prevention. Preprint at https://www.biorxiv.org/content/early/2017/11/09/162594 (2017).

11. WHO. Immunization, Vaccines and Biologicals: Hepatitis B. https://www.who. int/immunization/diseases/hepatitisB/en/ (2018).

12. $\mathrm{WHO} \mid$ guidelines for the prevention, care and treatment of persons with chronic hepatitis B infection. 2015. http://www.who.int/hiv/pub/hepatitis/ hepatitis-b-guidelines/en/.

13. Chang $\mathrm{M}-\mathrm{H}$. Breakthrough HBV infection in vaccinated children in Taiwan: surveillance for HBV mutants. Antivir Ther. 2010;15(3 Part B):463-9.

14. Pan $C Q$, et al. Tenofovir to prevent Hepatitis $B$ transmission in mothers with high viral load. N Engl J Med. 2016:374(24):2324-34.

15. Hyun $\mathrm{MH}$, et al. Systematic review with meta-analysis: the efficacy and safety of tenofovir to prevent mother-to-child transmission of hepatitis B virus. Aliment Pharmacol Ther. 2017;45(12):1493-505.

16. Chen J-Z, et al. Efficacy and safety of tenofovir disoproxil fumarate in preventing vertical transmission of hepatitis $B$ in pregnancies with high viral load. Sci Rep. 2017:7(1):4132.

17. Terrault NA, et al. Update on Prevention, Diagnosis, and Treatment of Chronic Hepatitis B: AASLD 2018 Hepatitis B Guidance. Hepatology. 2018; 67(4):1560-99.

18. Zampino R, et al. Hepatitis B virus burden in developing countries. World J Gastroenterol. 2015;21(42):11941-53.

19. Matthews PC, Geretti AM, Goulder PJR, Klenerman P. Epidemiology and impact of HIV coinfection with hepatitis B and hepatitis $C$ viruses in subSaharan Africa. J Clin Virol. 2014;61(1):20-33.

20. Andersson Ml, et al. Mother-to-child transmission of hepatitis B virus in subSaharan Africa: time to act. Lancet Glob Heal. 2015;3(7):e358-9.

21. Chotun $\mathrm{N}$, et al. Point-of-care screening for hepatitis $B$ virus infection in pregnant women at an antenatal clinic: a South African experience. PLoS One. 2017;12(7):e0181267.

22. Keane E, Funk AL, Shimakawa Y. Systematic review with meta-analysis: the risk of mother-to-child transmission of hepatitis B virus infection in subSaharan Africa. Aliment Pharmacol Ther. 2016;44(10):1005-17.
23. Wilson P, Parr JB, Jhaveri R, Meshnick SR. Call to action: prevention of mother-to-child transmission of Hepatitis B in Africa. J Infect Dis. 2018; 217(8):1180-3.

24. Njai HF, et al. Validation of rapid point-of-care (POC) tests for detection of Hepatitis B surface antigen in field and laboratory settings in the Gambia, Western Africa. J Clin Microbiol. 2015;53(4):1156-63.

25. Andersson MI, Maponga TG, ljaz S, Theron G, Preiser W, Tedder RS. High HBV viral loads in HIV-infected pregnant women at a tertiary hospital, South Africa. JAIDS J Acquir Immune Defic Syndr. 2012;60(4):e111-2.

26. Hoffmann CJ, et al. Maternal hepatitis B and infant infection among pregnant women living with HIV in South Africa. J Int AIDS Soc. 2014;17(1): 18871.

27. Andersson MI, et al. The epidemiology of hepatitis B virus infection in HIVinfected and HIV-uninfected pregnant women in the Western cape, South Africa. Vaccine. 2013;31(47):5579-84

28. Nachega JB, et al. Adherence to antiretroviral therapy during and after pregnancy in low-income, middle-income, and high-income countries: a systematic review and meta-analysis. AIDS. 2012;26(16):2039-52.

29. South Africa National Health Laboratory Service (NHLS) and Department of Health price list. (2015).

30. International Medical Products Price Guide. http://mshpriceguide.org (2015)

31. Beloukas A, Geretti AM. Hepatitis B virus drug resistance. In: Antimicrobial Drug Resistance. Cham: Springer International Publishing; 2017. p. 1227-42.

32. Hayer J, et al. HBVdb: a knowledge database for Hepatitis B Virus. Nucleic Acids Res. 2013;41:D566-D570. https://hbvdb.ibcp.fr/HBVdb/ (accessed on 18th April 2018)

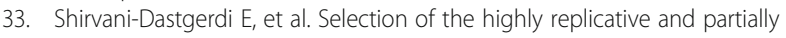
multidrug resistant rtS78T HBV polymerase mutation during TDF-ETV combination therapy. J Hepatol. 2017;67(2):246-54.

34. O'Hara GA, et al. Hepatitis B virus infection as a neglected tropical disease. PLoS Negl Trop Dis. 2017;11(10):e0005842.

35. Wang W, Wang J, Dang S, Zhuang G. Cost-effectiveness of antiviral therapy during late pregnancy to prevent perinatal transmission of hepatitis B virus. PeerJ. 2016:4:e1709.

36. Fan L, Owusu-Edusei K, Schillie SF, Murphy TV. Cost-effectiveness of activepassive prophylaxis and antiviral prophylaxis during pregnancy to prevent perinatal hepatitis B virus infection. Hepatology. 2016:63(5):1471-80.

37. Jourdain $\mathrm{G}$, et al. Tenofovir versus placebo to prevent perinatal transmission of Hepatitis B. N Engl J Med. 2018:378(10):911-23.

38. Mokaya J, et al. A systematic review of hepatitis B virus (HBV) drug and vaccine escape mutations in Africa: a call for urgent action. PLoS Negl Trop Dis. 2018;12(8):e0006629.

39. Easterbrook P, et al. HIV and Hepatitis Testing: Global Progress, Challenges, and Future Directions. AIDS Rev. 2016;18(1):3-14.40.

40. Spearman CW, et al. Hepatitis B in sub-Saharan Africa: strategies to achieve the 2030 elimination targets. Lancet Gastroenterol Hepatol. 2017:(12):900-9.

41. MCNaughton A, et al. HBV vaccination and PMTCT as elimination tools in the presence of HIV: insights from a clinical cohort and dynamic model.BMC Med. 2019;17(1):43.

42. WHO. Consolidated guidelines on the use of antiretroviral drugs for treating and preventing HIV infection: Recommendations for a public health approach - Second edition. 2016. https://www.who.int/hiv/pub/arv/arv2016/en/. ISBN: 9789241549684

43. Lemoine M, \& Thursz MR. Battlefield against hepatitis B infection and HCC in Africa. Journal of Hepatology. 2017:66:645-654.

\section{Publisher's Note}

Springer Nature remains neutral with regard to jurisdictional claims in published maps and institutional affiliations. 